\title{
A Remark on the Hopf invariant for Spherical 4-braids
}

Akhmet'ev PM*

Professor in IZMIRAN, Troitsk, Moscow region, Russia

\author{
Abstract \\ An approach by J.Wu describes homotopy groups $\pi_{n}\left(\mathrm{~S}^{2}\right)$ of the standard 2-sphere as isotopy classes of spherical \\ $\mathrm{n}+1$--strand Brunnian braids. The case $\mathrm{n}=3$ is investigated for applications.
}

\section{Introduction}

An approach by Wu describes homotopy groups $\pi_{n}\left(S^{2}\right)$ of the standard 2-sphere as isotopy classes of spherical $n+1$-strand Brunnian braids, for more details, Theorem 1.2. This straightforward approach is not possible for $n=3$, i.e. for 4 -strand braids the connection with $\pi_{3}\left(S^{2}\right)$ was unknown.

The homotopy group $\pi_{3}\left(S^{2}\right)$ in an infinite cyclic group, detected by the Hopf invariant

$$
H: \pi_{3}\left(S^{2}\right) \mapsto \mathbb{Z}
$$

An element of $\pi_{3}\left(S^{2}\right)$ is represented by a mapping $h: S^{3} \mapsto S^{2}$, which is considered up to homotopy. The Hopf invariant $H(h)$ is welldefined as the integer linking number of two oriented curves $h^{-1}(a)$, $h^{-1}(b)$, where $\mathrm{a}, \mathrm{b} \in S^{2}$ be a pair of regular points of $h$. The Hopf invariant is very important for applications.

Proposition 7.1.1, sequence (17) gets an exact sequence, which algebraically describes the group Brunn ${ }_{4}$ of 4-straight Brunnian braids [1]. The key point of our elementary geometrical construction is to construct an alternative epimorphism onto the group $\mathbb{Z} \times \mathbb{Z}$, see Definition. The kernel of this epimorphism is a well-defined subgroup $B_{B r u} n_{4} \subset \mathrm{Br}_{4}$ of Brunnian braids in a new sense (let us remark that Brunn $_{4}$ is not a subgroup of Brun . Define the Hopf invariant as a

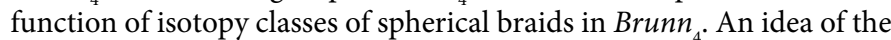
construction was coming from Graham and Roman [2]. However, the results by Ellis and Mikhailov are not adopted for physical applications.

The Hamiltonian provides an elegant method for generating simple geometrical examples of complicated braids and links, as is presented in Mitchell A Berger [3].

The paper is motivated by the following problems:

- Derive applications of higher-order winding numbers to generate turbulent motions of vortices in two dimensions. For a special Hamiltonian motion of 3 vortices on the plane this is done in Mitchell A Berger [3]. (Problem 1).

- To unify the approach Ch.3 to $\pi^{*}\left(S^{2}\right)$ with the Wu's approach (Problem 2) [4].

Let us clarify Problem 2. Let $\mathrm{F}$ be the space of functions $f: \mathbb{R}^{1} \rightarrow \mathbb{R}^{1}$ with "right" boundary conditions at the infinity. The derivative of the order 1,2 , and 3 of a function $f \in F$ can nowhere be vanished simultaneously. Define the mapping, $A: F \rightarrow \Omega\left(\mathbb{R}^{3} \backslash 0\right)$, by the formula $A(f)=\left\{x \mapsto\left(\frac{d f(x)}{d x}, \frac{d^{2} f(x)}{d x^{2}}, \frac{d^{3} f(x)}{d x^{3}}\right)\right\}$.

V.I.Arnold (1996) conjectured that the induced homomorphism $A_{n}: \pi_{n}(F) \rightarrow \pi_{n}\left(\Omega\left(S^{2}\right)\right) \cong \pi_{n}+1\left(S^{2}\right)$ is an isomorphism for $n \geq 0$. This theorem was proved by V.A. Vassiliev in the special case $n+2$, and by
Eliashberg and Mishachev in the general case.

The paper is organized as following. In Section 2 we recall required definitions concerning first-order stage of the construction and determine the linking numbers of spherical 4-component braids. In Section 3 the Hopf invariant for 4-component spherical braids is defined. This is a second-order particular defined invariant: to define this invariant we should assume that the all linking numbers (there are two) of components of a spherical braid are equal to zero. Results are formulated in Theorems 4, 6. The main result is the Corollary 8. In Section 4 we give proofs.

\section{A possible application for turbulences (Problem 1)}

Assume a motion of a large collection of $n$ vortexes (or, particles) in a bounded domain $U$ on the plane is investigated. The trajectories of vortexes (or, of particles) in the configuration space, i.e. in the Cartesian product, of the domain and the time, are represented by a braid $F$, components of the braid $F$ correspond to vortexes in the collection. Assume that the windings numbers of components of the braid $F$ are distributed as in the statement of Corollary. This means that the length of the segment $(\mathrm{a}, \mathrm{b})$, which is assumed sufficiently large, is bounded from below; the upper bound depends of the number $n$ of vortexes in the collection. We may replace $F$ by a colored braid, if $b-a$ is sufficiently large, using the Arnol'd collection of the short paths, we have no loss of a generality.

Otherwise, assume that the bound $k$ of the distribution of full angles of windings numbers is much less then the number $n$ of partials. Consider the normalized sum of squares of Hopf invariants

$$
\Upsilon=\frac{N}{(b-a) l} \sum_{i=1}^{l} H^{2}\left(g_{i}\right), \quad N=C_{n}^{4}=\frac{n^{4}}{24}+O\left(n^{3}\right)
$$

this sum is taken over all collection of admissible quadruples of components of $F$, the number $l$ of admissible quadruples could be sufficiently large by Corollary 8 . The following statements will be proved, or disproved, elsewhere:

- $\Upsilon$ is the universal constant of the motion, which depends no of the time scale and of the time interval [a, b] itself;

- The constant $Y$ is large (correspondingly, is small), if the motion of

*Corresponding author: Dr. Akhmet'ev PM, IZMIRAN, Troitsk, Moscow region, Russia, Tel: +7(4967) 510912; Fax: +7(495)3340124; E-mail: pmakhmet@mi.ras.ru

Received October 31, 2013; Accepted January 28, 2014; Published March 10, 2014

Citation: Akhmet'ev PM (2014) A Remark on the Hopf invariant for Spherical 4-braids. J Phys Math 5: 124 doi: 10.41722090-0902.1000124

Copyright: ( 2014 Akhmet'ev PM. This is an open-access article distributed under the terms of the Creative Commons Attribution License, which permits unrestricted use, distribution, and reproduction in any medium, provided the original author and source are credited. 
the system of vortexes (or, of partials) is turbulent (correspondingly, the system is closed to an integrable system);

- Assume the sum (2) is taken over all admissible quadruples, between which the distance is smaller then $L$. Then $\Upsilon(L)$ correlates with the spacial turbulent spectra of the motion up to the scale $L$.

\section{Linking Numbers For Spherical Braids}

By a spherical (ordered) $n$-braid we mean a collection of embeddings of the standard circles

$$
f: \bigcup_{i=1}^{n} S_{i}^{1} \subset S^{2} \times S^{1}
$$

where the composition of this embedding with the standard projection $S^{2} \times S^{1} \rightarrow S^{1}$ on the second factor in the target space, restricted to an arbitrary component $S_{i}^{1}, i=1, \ldots, n$ is the identity mapping.

The set of all ordered spherical n-braids up to isotopy is denoted by $\mathrm{Br}_{n}$. It is well-known that $\mathrm{Br}_{n}$ is a group.

For a fixed value $t \in S^{1}$, a braid $\mathrm{f} \in B r_{n}$ intersects the level $S^{2} \times t$ by an (ordered) collection of $n$ points $\left\{Z_{1}(t), \ldots Z_{n}(t)\right\}$. Let assume that $n=4$. Denote by

$$
g=g(f): S_{1}^{1} \cup S_{2}^{1} \cup S_{3}^{1} \subset S^{2} \times S^{1},
$$

the 3-component braid, obtained from $f$ by eliminating of the last component $S_{4}^{1}$.

Let us identify the sphere $S^{2}$ with the Riemann sphere, or with the complex projective line $C$. For a braid $f$ let us consider the collection of Mobius transformations, which transforms the points $\mathrm{z}_{1}, \mathrm{z}_{2}, \mathrm{z}_{3}$ into 0 , $1, \infty$ correspondingly:

$$
F(z ; t)=\frac{\left(z-z_{1}(t)\right)\left(z_{2}(t)-z_{3}(t)\right)}{\left(z-z_{3}(t)\right)\left(z_{2}(t)-z_{1}(t)\right)} .
$$

The image $F(f)$ is a 4 -strand braid with the constant components $\left\{\mathrm{z}_{1}(t), \mathrm{z}_{2}(t), \mathrm{z}_{3}(t)\right\}=\{0,1, \infty\}$. Denote this braid by

$F(f)=f^{\text {norm }}$.

The 3-strand braid g, constructed from frorm is the constant in the points $\{0,1, \infty\}$. The last component fiorm $\left(S^{1}\right)$ of $F(f)$ is represented by a closed path $z_{4}(t) \in \widehat{\mathbb{C}} \backslash\{0,1, \infty\}, t \in \mathbb{R}^{1} / 2 \pi$. Note that, generally speaking, braids $f$, frorm are not isotopic. Moreover, if $f$ is a Brunnian in the sense [2], fiorm is, generally speaking, not a Brunnian.

For a given (ordered) 4-component braid $f$ let us define the linking number $L k(f)$,

$$
L k: \mathrm{Br}_{4} \rightarrow \mathbb{Z} \text {. }
$$

Consider the following 1-form

$\omega_{0}=\frac{1}{2 \pi i} \frac{d z}{z}$.

By definition we get

$$
d \log (z)=\frac{1}{2 \pi i} \frac{d z}{z},
$$

where $\log (\mathrm{z})$ is given by the formula:

$$
\log (z)=(2 \pi i)^{-1} \int \frac{d z}{z},
$$

assuming that $\log (1)=0$, as a multivalued complex function.

Define $L k(f)$ by the formula:

$$
L k(f)=\Re \int_{0}^{2 \pi} \frac{d z_{4}(t)}{z_{4}(t)}=\int_{f_{4}^{n o r m}} \omega_{0},
$$

where $\mathfrak{R}$ is the real part of the integral. By construction, $L k(f)$ is the winding number, i.e. the integer number of rotations of the path $\mathrm{z}_{4}(t)$ of frorm with respect to the origin and the infinity in $C$.

The permutation group $\Sigma(4)$ of the order 24 acts on the space of ordered spherical braids:

$$
\Sigma(4) \times \mathrm{Br}_{4} \rightarrow \mathrm{Br}_{4} .
$$

The image of an ordered braid $f$ by a transposition $\sigma:(1,2,3,4) \mapsto\left(\sigma_{1}, \sigma_{2}, \sigma_{3}, \sigma_{4}\right)$ is well-defined by the corresponding re-ordering of components of $f$. Let us investigate the orbit of the linking numbers $L k(f)$ with respect to (7). Simply say, we investigate how many independent linking numbers of components of braids are well-defined?

Let us consider the following exact sequences of groups:

$$
\begin{aligned}
& 0 \rightarrow A_{4} \rightarrow \Sigma_{4} \stackrel{\text { sign }}{\rightarrow} \mathbb{Z} / 2 \rightarrow 0, \\
& 0 \rightarrow \mathbb{Z} / 2 \times \mathbb{Z} / 2 \rightarrow A_{4} \rightarrow \mathbb{Z} / 3 \rightarrow 0 .
\end{aligned}
$$

The subgroup $A_{4} \subset \Sigma_{4}$ in the sequence (8) is represented by permutations, which preserve signs (equivalently, which is decomposed into an even number of elementary transpositions). The subgroup $\mathbb{Z} / 2 \times \mathbb{Z} / 2 \subset A_{4}$ in the sequence (9) is generated by the permutations $\{(1,2)(3,4) ;(1,3)(2,4) ;(1,4)(2,3)\}$.

Let us consider 2-primary subgroup $\mathrm{K} \subset \Sigma_{4}$ (the dihedral group of the order 8), which is defined as the extension of the subgroup $\mathbb{Z} / 2 \times \mathbb{Z} / 2$ from the sequence (9), which is included in the sequence (8). An epimorphism

$$
\theta=\left(\theta_{1}, \theta_{2}\right): K \rightarrow \mathbb{Z} / 2 \times \mathbb{Z} / 2,
$$

is defined as follows: $\theta_{1}(\sigma)=1$ (the group $Z / 2$ is in the multiplicative form), if $\sigma$ preserves a (non-ordered) partition $(1,3)(2,4)$, and $\theta_{1}(\sigma)$, and $\theta_{1}(\sigma)=-1$, otherwise. Therefore $\theta_{1}$ is an epimorphism with the kernel $\mathbb{Z} / 2 \times \mathbb{Z} / 2$ from the left subgroup of the sequence (9). The epimorpism $\theta_{2}(\sigma)$ is determined by the sign of a permutation $\sigma$, this is the restriction of the right epimorphism in the sequence (8) to the subgroup $K \subset \Sigma_{4}$. The kernel $\operatorname{Ker}(\theta) \cong \mathbb{Z} / 2$ is the center of the dihedral group $K$.

\section{Lemma 1}

1. The function (4) is invariant with respect to the action (7) by an arbitrary permutation, which in the kernel of $\theta$ in (10).

2. The function (4) is skew-invariant with respect to the action by a permutation, which is in the kernel of $\theta_{1}$ (the composition of $\theta$ with the projection on the first factor, but not in the kernel of $\theta_{2}$ (the composition of $\theta$ with the projection on the second factor).

3. Denote by $\tilde{f} \in B r_{4}$ the ordered braid, which is obtained from $f \in \mathrm{Br}_{4}$ by the action (7) by the element $(1,2)$ is the product of the generators of the factors). There exists an ordered braids $f \in B r_{4}$, for which the linking numbers $L k(f), L k(\tilde{f})$ are arbitrary integers.

From Lemma one may deduce the following corollary.

\section{Corollary 2}

1. For an arbitrary braid $f \subset B r_{4}$ the linking number $L k(f)$, is welldefined as the differences of the winding number of the component 2 between the components 1 and 3 with the winding number of the component 4 between the components 1 and 3 .

2. For a braid, where $f \in \mathrm{Br}_{4}$ is an arbitrary, $\tilde{f}$ is defined in Lemma 
1, the linking number $\operatorname{Lk}(\tilde{f})$ is well-defined as the difference of the winding number of the component 2 between the components 1 and 3 with the winding number of the component 4 between the components 2 and 3.

Corollary (2) motivates the following definition.

\section{Definition 3}

Let $f \in \mathrm{Br}_{4}$ be a (ordered) spherical braid. Define the total linking number $L K(f) \in \mathbb{Z} \oplus \mathbb{Z}$ by the following formula:

The total linking number is a well-defined homomorphism

$$
L K: B r_{4} \mapsto \mathbb{Z} \oplus \mathbb{Z}
$$

\section{Hopf Invariant of Braids}

Let $f \in \mathrm{Br}_{4}$ be a (ordered) spherical braid with the trivial total linking number: $L k(f)=0$. Such braids generate the subgroup in the group $\mathrm{Br}_{4}$, denote this subgroup by $\mathrm{Brunn}_{4} \subset \mathrm{Br}_{4}$. Let us remark that this subgroup does not coincide with the subgroup of Brunnian braids Brun $_{4}$, defined in Berrick et al. [1], Theorem 1.2.

\section{Theorem 4}

There exists a well-defined homomorphism

$H:$ Brunn $_{4} \rightarrow Z$,

called the Hopf invariant. The homomorphism (11) is invariant with respect to the action (7) by an arbitrary permutation, which in the kernel of $\theta_{2}$ in (10) (this homomorphism is defined as the sign of a permutation of straights), and is skew-invariant with respect to the action by a permutation, which is not in the kernel of $\theta_{2}$.

\section{Definition of the hopf invariant}

In this section we present the construction, which is closed to Theorem 3 of Mitchell A Berger [3], using differential topology instead of homology algebra. Let $\mathrm{f} \in \mathrm{Brunn}_{4}$ be an arbitrary. Consider the braid $f^{\text {norm }}$, given by Mitchell A Berger [3]. Recall, the braid $g \in B r_{3}$, which consists of the straits (1-3) of frorm, is the constant braid at the points 0 , $1, \infty$ in correspondingly. Consider the strait (4) of the braid form. This strait is represented by an oriented closed path $z_{4}: S^{1} \rightarrow \hat{C} \backslash\{0 \cup 1 \cup \infty\}$. This path determines a cycle, which is an oriented boundary, because of the condition $L K\left(\right.$ frorm $^{\text {n }}=0$.

Let us prove that $L k(f)=0$. Denote the group of Mobius transformations by $M$. The standard inclusion $\mathrm{SO}(3) \subset \mathrm{M}$ is welldefined. This inclusion is a homotopy equivalence, therefore we get $\pi_{1}(M)=\pi_{1}\left(S O(3)=\mathbb{Z} / 2\right.$. This proves that $\operatorname{LK}\left(2 f^{\text {horm }}\right)=\mathrm{LK}(2 \mathrm{f})$. Because $\operatorname{LK}\left(2 f^{\text {form }}\right)=2 \operatorname{LK}\left(2 f^{\text {form }}\right), \operatorname{LK}(2 \mathrm{f})=2 \operatorname{Lk}(f)$, we get $\operatorname{LK}\left(f^{\text {norm }}\right)=L k(f)$. The equality $L k(f)=0$ is proved.

Consider the inclusions

$$
\begin{aligned}
& I_{0}: C \backslash\{0 \cup 1 \cup \infty\} \subset C \backslash\{1 \cup \infty\}, \\
& I_{\infty}: C \backslash\{0 \cup 1 \cup \infty\} \subset C \backslash\{0 \cup 1\}, \\
& I_{1}: C \backslash\{0 \cup 1 \cup \infty\} \subset C \backslash\{0 \cup \infty\} .
\end{aligned}
$$

Because $\quad H_{1}(\hat{\mathbb{C}} \backslash\{1 \cup \infty\} ; \mathbb{Z})=\pi_{1}(\hat{\mathbb{C}} \backslash\{1 \cup \infty\})=\mathbb{Z}, \quad$ the condition $\mathrm{LK}($ frorm $)=0$ implies $I_{0}, \#([i])=0$, for the homomorphism

$$
I_{0, \#}: \pi_{1}(C \backslash\{0 \cup 1 \cup \infty\}) \rightarrow \pi_{1}(C \backslash\{0 \cup \infty\}) .
$$

Analogously $I_{\infty, \#}([i])=0, I_{1}, \#([i])=0$.

There exist the following 3 maps of the standard 2-disk

$$
\begin{array}{ll}
e_{0}: D_{0}^{2} \rightarrow \hat{C} \backslash\{1 \cup \infty\}, & \left.e_{0}\right|_{\partial D^{2}}=z_{4}, \\
e_{\infty}: D_{\infty}^{2} \rightarrow \hat{C} \backslash\{0 \cup 1\}, & \left.e_{\infty}\right|_{\partial D^{2}}=z_{4}, \\
e_{1}: D_{1}^{2} \rightarrow \hat{C} \backslash\{0 \cup \infty\}, & \left.e_{1}\right|_{\partial D^{2}}=z_{4} .
\end{array}
$$

Consider a 2-sphere, which is represented by a gluing $D^{2} \cup D^{2}$ of the disks $D_{0}^{2} \cup D_{\infty}^{2}$ along the common boundary, which is identified with the circle $S_{4}^{1}$. Denote this sphere by $S_{1}^{2}$. Analogously define spheres $S_{0}^{2}=D_{\infty}^{2} \cup_{\partial} D_{1}^{2}, S_{\infty}^{2}=D_{1}^{2} \cup_{\partial} D_{0}^{2}$. Because the target spaces of the mappings $e_{0}, e_{\infty}, e_{1}$ are aspherical, the corresponding mapping is well-defined up to homotopy.

Consider the following commutative diagram of inclusions:

$$
\begin{array}{ccc}
C \backslash\{0 \cup \infty \cup 1\} & \subset & C \backslash\{0 \cup \infty\} \\
\cap & \cap \\
C \backslash\{\infty \cup 1\} & \subset & C \backslash\{\infty\}
\end{array}
$$

Consider the mappings $e_{0}: D_{0}^{2} \rightarrow C \backslash\{1 \cup \infty\}, e_{1}: D_{1}^{2} \rightarrow C \backslash\{0 \cup \infty\}$ to the left bottom and to the right upper spaces of the diagram (12) correspondingly. The mapping $e_{0} \cup_{\hat{\partial}} e_{1}: S_{\infty}^{2} \rightarrow C \backslash\{\infty\}$ is well defined by gluing of the two mappings $e_{0}, e_{1}$ along the common mapping $i$ of the boundaries. Consider the standard 3-ball $D_{\infty}^{3}$ (with corners along the curve $S_{4}^{1}$ ) with the boundary $\partial D_{\infty}^{3}=S_{\infty}^{2}$. The mapping $e_{0} \cup \partial e_{1}$ can be extended to the mapping

$$
d_{\infty}: D_{\infty}^{3} \rightarrow \hat{C} \backslash\{\infty\} .
$$

The target space of this mapping is the right bottom space of the diagram (12). Because the target space of the mapping $d_{\infty}$ is contractible, the mapping $d_{\infty}$ is well-defined up to homotopy. By the analogous constructions the following mappings

$$
\begin{aligned}
& d_{1}: D_{1}^{3} \rightarrow \hat{\mathbb{C}} \backslash\{1\}, \\
& d_{0}: D_{0}^{3} \rightarrow \hat{\mathbb{C}} \backslash\{0\}
\end{aligned}
$$

are well-defined.

The mappings (13), (14), (15) determine the mapping

$$
h=h(f): S^{3} \rightarrow S^{2}
$$

as follows. Take a 3-sphere $S^{3}$, which is catted into 3 balls $D_{\infty}^{3}, D_{1}^{3}, D_{0}^{3}$ along the common circle $S_{4}^{1} \subset S^{3}$. The sphere $S^{3}$ is represented as the join $S_{4}^{1} * S_{a}^{1}$ of the two standard circle. On the circle $S_{a}^{1}$ take 3 points $x_{0}, x_{1}, x_{\infty} \in S_{a}^{1}$ The subsets $S_{4}^{1} *\left[x_{0}, x_{1}\right] \subset S^{3}, S_{4}^{1} *\left[x_{1}, x_{\infty}\right] \subset S^{3}, S_{4}^{1} *\left[x_{\infty}, x_{0}\right] \subset S^{3}$ are 3 copies of $3 \mathrm{D}$ disks, which are glued along corresponding subdomains in its boundaries.

Let usidentify $D_{\infty}^{3} \cong S_{4}^{1} *\left[x_{0}, x_{1}\right], D_{0}^{3} \cong S_{4}^{1} *\left[x_{1}, x_{\infty}\right], D_{1}^{3} \cong S_{4}^{1} *\left[x_{\infty}, x_{0}\right]$. The boundary $\partial D_{\infty}^{3}$ is identified with the balls $S_{4}^{1} *\{0\} \cong D_{0}^{2}, S_{4}^{1} *\{1\} \cong D_{1}^{2}$, which are glued along the common boundary $S_{4}^{1}$. The boundary $\partial D_{0}^{3}$ is identified with the balls $S_{4}^{1} *\{1\} \cong D_{1}^{2}, S_{4}^{1} *\{\infty\} \cong D_{\infty}^{2}$, which are identify along the common boundary $S_{4}^{1}$. The boundary $\partial D_{1}^{3}$ is identified with the balls $S_{4}^{1} *\{\infty\} \cong D_{\infty}^{2}, S_{4}^{1} *\{0\} \cong D_{0}^{2}$, which are identified along the same boundary $S_{4}^{1}$. The mappings $d_{0}, d_{1}, d_{\infty}$ on the corresponded balls are well-defined by the formulas (13-15) correspondingly. This mappings define the mapping (16) on the 3 -sphere.

Definition 5: The Hopf invarian $H(f)$ for a braid $f \in B_{\text {Brunn }}$ in the formula (11) is defined as the Hopf invariant of the mapping $h$ by the formula (1). The mapping $h=h(f)$ is explicitly defined from the braid $f$ by the formula (16). 


\section{A formula to calculate the Hopf invariant}

Let us introduces an explicit formula to calculate the Hopf invariant for a braid $\mathrm{f} \in \mathrm{Brunn}_{4}$. Consider the complex plane C. The 4-th strain of the braid fiorm determines a curve on the plane without two points $\{0.1\}$, which is denoted by

$$
\gamma: S^{1} \rightarrow \mathbb{C} \backslash\{0 \cup 1\} .
$$

Let us consider the closed 1-form (4). Define a complex 1-form

$$
\omega_{1}=\frac{1}{2 \pi i} \frac{d z}{z-1} \text {. }
$$

Define a real (multivalued) function $\lambda_{0}$ by integration along the path $\gamma(t), t \in[0, t] \subset S^{1}$ of the real part of the form (18) as following:

$$
\lambda_{0}(t)=\Re \int_{0}^{t} \omega_{0} .
$$

Define a real (multivalued) function $\lambda_{1}$ by integration along the path of the real part of the form (17) as following:

$$
\lambda_{1}(t)=\Re \int_{0}^{t} \omega_{1} \text {. }
$$

To take the multivalued functions (18), (19) well-defined, assume that the path $\gamma$ starts at the point $2 \in \mathbb{C}: \lambda_{0}(0)=2, \lambda_{1}(0)=2$.

Define a closed 1-form $\Psi(t)$ along a curve $\gamma(t) \in \mathbb{C} \backslash\{0 \cup 1\}$ by the following formula:

$$
\psi(t)=\lambda_{0}(t) \omega_{1}+\lambda_{1}(t) \omega_{0}
$$

Let us consider a function, which is well-defined as the real part of the integral

$$
\Psi(T)=\Re \int_{0}^{T} \psi(t) d \gamma, \quad t \in[0, T] \subset S^{1}, \Psi(0)=0 .
$$

\section{Theorem 6}

The Hopf invariant of a braid $f \in$ Brunn $_{4}$ in the formula (11), which is defined by Definition 5, is calculated by the formula:

$$
-H(f)=\Psi(2 \pi)=\frac{1}{2} \mathfrak{R} \int_{0}^{2 \pi} \psi(t) d \gamma,
$$

where $\gamma$ is the closed path, determined by the 4 -th straight of the braid $f^{\text {norm }}$ by the formula (17).

From Theorem 6 we get a corollary.

\section{Corollary 7}

1. The Hopf invariant (11) is an epimorphism.

2. Assume there is a braid $f \in B_{r u n n}$ for which the braid frorm is represented by a commutator of the straight (4) with straights (1) and (2) (such a braid is called the Borromean rings). Then $H(f)= \pm 1$, where the sign in the formula depends on the sign of the commutator.

\section{Proof of corollary}

It is sufficient to prove --2. The right-hand side of the formula (21) coincides with the formula (28) [1], which is simplified for the considered example. The Berger's formula is applied for the 3-uple configuration space, this gives the opposite sign for the last term in the formula (21) with respect to the origin formula. For the Borromean ring the formula (22) is non-trivial. The right side of the formula gives $H(f)=1$ for the right Borromean rings. Corollary is proved.

The following Corollary is the main result of the paper. The author hope that this result is the initial step toward the solution of the first problem, mentioned in Introduction.

\section{Corollary 8}

Assume we have a classical -conponent colored non-ordered braid $F$, $n>>4$, for which all pairwise winding (integer) numbers of components are distributed to the segment: $\{-2 k \pi, \ldots-2 \pi, 0,2 \pi, \ldots 2 k \pi\}, 0<k<<n$. Let $G$ is the spherical braid, which is defined as the image of $F$ by the stereography projection $\mathbb{R}^{2} \times I \rightarrow S^{2} \times I$. Then there exist at least $K=\frac{n^{4}}{24(2 k+1)^{2}}+O\left(n^{3}\right) \quad(K(n) \rightarrow+\infty$ when $n \rightarrow+\infty) \quad$ 4-component subbraids $f i \subset F$, for which $L K\left(g_{i}\right)=0, g_{i} \subset G$. In particular, the squares $H^{2}\left(g_{i}\right) \in \mathbb{N}$ are well-defined.

\section{Proof of corollary}

Proof is evident: the number $K$ of subbraids $g_{i} \subset F$ with trivial total linking number $L K\left(g_{i}\right)=0$ is explicitly estimated from below using integers $k, n$.

\section{Proofs}

\section{Proof of lemma}

Proof of Statement 1. Take an oriented 3--manifold $M^{3}$. Take two disjoin oriented cycles $C_{I} \subset M^{3}, C_{I I} \subset M_{3}$, which represent the trivial homology class

$$
0=\left[C_{I}\right]=\left[C_{I I}\right] \in H_{1}\left(M^{3} ; Z\right) .
$$

The linking number link $\left(L_{P}, L_{I I}\right)$ is a well-defined integer the algebraic intersection coefficient of the boundary $\left(\Gamma_{I}, \partial \Gamma_{I}\right) \subset M^{3}$, $\partial \Gamma_{I}=C_{I}$. The linking number $\operatorname{link}\left(C_{P} C_{I I}\right)$ is well defined, because of the condition.

Obviously, link $\left(C_{T}, C_{I I}\right)=$ link $\left(C_{I I}, C_{T}\right)$, because the collections of signed points $A_{I}=\Gamma_{I} \cap C_{I I}$ and $A_{I I}=C_{I} \cap \Gamma_{I I}$ represent the same cycle $\left[A_{I}\right]=\left[A_{I I}\right] \in H_{0}\left(M^{3} ; Z\right)$. The boundary of $-\left[A_{I}\right] \cup\left[A_{I I}\right]$ is given by the oriented curve $\Gamma_{I} \cap \Gamma_{I I}$.

Take $M^{3}=S^{2} \times S^{1}$. Take an arbitrary braid fiorm. The cycle $C_{I}$ is represented by the images of the following two closed paths $\left[z_{1}(t)\right]=0$ $x-S^{1},\left[z_{3}(t)\right]=\infty \times S^{1}, t \in[0,2 \pi]$, where the path $z_{1}(t)$ is taken with the opposite orientation along $S^{1}$. The cycle $C_{I I}$ is represented by the two closed paths $\left[z_{2}(t)\right]=0 \times-S^{1},[\mathrm{z} 4(\mathrm{t})] \subset S^{2} \times S^{1}$, where the path $z_{2}(t)$ is taken with the opposite orientation along $S^{1}$.

Take $\sigma_{\alpha}=(1,2)(3,4), \sigma_{\alpha}$ is the generator of $\operatorname{Ker}(\theta)$. It is easy to see that $\operatorname{Lk}\left(f^{\text {norm }}\right)=\operatorname{link}\left(C_{I}, C_{I I}\right), \operatorname{Lk}\left(\sigma_{\alpha} \times f^{\text {norm }}\right)=\operatorname{link}\left(C_{I I}, C_{I}\right)$. Statement 1 is proved.

Proof of Statement 2. Assume $\sigma_{b}=(1,3)$, the case $\sigma_{b}=(2,4)$ is analogous. Then $\operatorname{Lk}\left(f^{\text {form }}\right)=\operatorname{link}\left(C_{I}, C_{I I}\right), L k\left(\sigma \mathrm{b} \times f^{\text {form }}\right)=\operatorname{link}\left(-C_{P}, C_{I I}\right)$. Therefore we get $L k\left(f^{\text {form }}\right)=-L k\left(\sigma \mathrm{b} \times f^{\text {horm }}\right)$. Statement 2 is proved.

Lemma 1 is proved.

\section{Proof of Corollary 2}

Statements 1,2 are obvious.

Proof of Statement 3. The straights $\left\{z_{1}(t), z_{2}(t), z_{3}(t), z_{4}(t)\right\}$ determines 6 pairs of -cycles in $S^{2} \times S^{1}$. A function of winding numbers of component is given by a linear combinations of linking numbers between the corresponding pairs of cycles. To prove that such a function is well-defined, we have to assume that the each cycle is a boundary. Denote the cycle, generated by the pair of paths $-z$ $(t), z_{j}(t)$ by $C_{i, j}$. We have the following identity: link $\left(C_{1,2}, C_{3,4}\right)+$ link $\left(C_{2,3}, C_{14}\right)+$ link $\left(C_{3,1}, C_{2,4}\right)=0$, and the analogous 3 identities, which are obtained by the permutation of the indexes. Therefore we get a 
collection of 2 independent well-defined linking numbers. Statement is proved. Corollary 2 is proved.

\section{Proof of theorem}

Let us prove that the homomorphism (11) is skew-invariant with respect to the action (7) by an odd permutation. Assume that the permutation $\sigma$ is given by an elementary transposition of straights with number (1-3) say by the transposition $\sigma=(1,2)$. Then by the formula (16), the mappings $h(f)$ is related with the mapping $h(\sigma \times f)$ by the composition with the reflection $S^{3} \rightarrow S^{3}$, which translates the curve $S_{4}^{1} \subset S^{3}$ to itself, and permutes the points $x_{0}, x_{1}$ on the circle $S_{a}^{1}$. The reflection changes the homotopy class of $h$ to the opposite. This proves Theorem in this case.

Assume that $\sigma=(1,4)$ (the cases $\sigma=(2,4)$, or $(3,4)$ are analogous). Then we may calculate the Hopf invariants of the mappings $h(f)$ and $h(\sigma \times f)$, using the formula (22) (Theorem 6 is proved below). The Mobius group is locally contractible. Therefore, the ordered braid $(\sigma \times f)$ is isotope to the braid $f$ in which the components $(1,4)$ are renumbered. The restriction of the considered isotopy on the common straight at $\infty \in C$ is the identity.

By Statement 2 of Corollary 7, the Hopf invariant $h\left(\sigma \times f^{\text {form }}\right)$ is defined as the length of commutators of the straight (1) with straights (4) and (2).

The Hopf invariant for $h(f)$ coincides with the commutator of the straight (4) with the straights $(1,4)$. Therefore the Hopf invariant for $h(\sigma \times f)$ is opposite to the Hopf invariant for $h(f)$, because the sign of the commutator is changed by a permutation of components.

Theorem 4 is proved.

\section{Proof of Theorem 6}

Consider the mapping $h: S^{3} \rightarrow S^{2}=\hat{C}$, which is defined by the formula (16). Take two normalized volume forms $\Omega_{0}, \Omega_{1} \in \Lambda^{2}\left(S^{2}\right)$ :

$$
\iint_{S^{2}} \Omega_{0}=\iint_{S^{2}} \Omega_{1}=1
$$

The forms $\Omega_{0}, \Omega_{1}$ are defined as the standard ill-supported forms at the points 0,1 , correspondingly. The Hopf invariant (11) is calculated by the formula:

$$
H(f)=\frac{1}{2} \iiint_{S^{3}} h^{*}\left(\Omega_{0}\right) \wedge \beta_{1}+h^{*}\left(\Omega_{1}\right) \wedge \beta_{0},
$$

where $x \in S^{3}, h^{*}\left(\Omega_{0}\right) \in \Lambda^{2}\left(S^{3}\right)$ is the pull-back of $\Omega_{0} \in \Lambda^{2}\left(S^{2}\right)$ by $h$ : $S^{3} \rightarrow S^{2}, \beta_{0} \in \Lambda^{1}\left(S^{3}\right)$ is an arbitrary 1 -form, such that $d\left(\beta_{0}\right)=h^{\star}\left(\Omega_{0}\right)$, the 1 -form $\beta_{1} \in \Lambda^{1}\left(S^{3}\right)$ is defined analogously to $\beta_{0}$.

Evidently, the 1 -forms $\beta_{0}$ in the integral (24) is represented in its cohomology class by a cocycle, which satisfies the condition $h^{\star}\left(\Omega_{0}\right)=d \beta_{0}=0$ inside the ball $D_{0}^{3}$. This follows from the fact that the curve $h^{-1}(0)$ is outside the ball $D_{0}^{3}$. In the formula (24) the first term is well-defined up to gauge transformation $\beta_{0} \mapsto \beta_{0}+\operatorname{grad} \varphi_{0}$. We may put $\beta_{0}=0$ in $D_{0}^{3}$, and keep $\beta_{0}$ on $D_{1}^{2}=D_{0}^{3} \cap D_{\infty}^{3}$.

Analogously, $d \beta_{1}=0$ in the ball $D_{1}^{3}$. In the second term in the integral (24), using $\beta_{1} \mapsto \beta_{1}+\operatorname{grad} \varphi_{1}$, we get $\beta_{1}=0$ in $D_{1}^{3}$, and keep $\beta_{1}$ on $D_{0}^{2}$. Then we get the following simplification of (24):

$$
H(f)=\frac{1}{2} \iiint_{D_{\infty}^{3}} h^{*}\left(\Omega_{0}\right) \wedge \beta_{1}+h^{*}\left(\Omega_{1}\right) \wedge \beta_{0} .
$$

In the ball $D_{\infty}^{3}$ the 3 -form $h^{*}\left(\Omega_{0}\right) \wedge \beta_{1}$ is exact, we get $\alpha_{1} \in \Lambda^{2}\left(D_{\infty}^{3}\right)$, $d \alpha_{1}=h^{*}\left(\Omega_{0}\right) \wedge \beta_{1}$. Moreover, we may put $\alpha_{1}=\beta_{0} \wedge \beta_{1}$ over $D_{1}^{2}=D_{\infty}^{3} \cap D_{0}^{3}$, $\alpha_{0}=0$ over $D_{0}^{2}=D_{\infty}^{3} \cap D_{1}^{3}$.

In the ball $D_{\infty}^{3}$ the 3 -form $h^{*}\left(\Omega_{1}\right) \wedge \beta_{0}$ is exact, we get $\alpha_{0} \in \Lambda^{2}\left(D_{\infty}^{3}\right)$, $d \alpha_{0}=h^{*}\left(\Omega_{1}\right) \wedge \beta_{0}$. We may put $\alpha_{0}=\beta_{1} \wedge \beta_{0}=-\beta_{0} \wedge \beta_{1}$ over $D_{0}^{2}$, and $\alpha_{0}=0$ over $D_{1}^{2}$.

Apply the 3D Gauss-Ostrogradsky formula, we get

$$
\begin{aligned}
& \iiint_{D_{\infty}^{3}} h^{*}\left(\Omega_{0}\right) \wedge \beta_{1}=\iint_{D_{1}^{2}} \beta_{0} \wedge \beta_{1}, \\
& \iiint_{D_{\infty}^{3}} h^{*} \Omega_{1} \wedge \beta_{0}=-\iint_{D_{0}^{2}} \beta_{0} \wedge \beta_{1} .
\end{aligned}
$$

The 2 -form $\beta_{0} \wedge \beta_{1} \in \Lambda^{2}\left(D_{1}^{2}\right)$ is exact. Because in the disk $D_{1}^{2}$ the 0 -form $\lambda_{1}$ is well defined, and $d \lambda_{1}=\beta_{1}$, we get: $d\left(\lambda_{1} \beta_{0}\right)=-\beta_{0} \wedge \beta_{1}$.

Analogously, the 2 -form $\beta_{0} \wedge \beta_{1} \in \Lambda^{2}\left(D_{0}^{2}\right)$ is exact. Because in the disk $D_{0}^{2}$ the 0 -form $\lambda_{0}$ is well defined, and $d \lambda_{0}=\beta_{0}$, we get: $d\left(\lambda_{0} \beta_{1}\right)=\beta_{0} \wedge \beta_{1}$.

Apply the 2D Green formula (singular points of $\beta_{0}, \beta_{1}$ give no contribution to the integral over the boundary) we get:

$$
\begin{aligned}
& \iint_{D_{1}^{2}} \beta_{0} \wedge \beta_{1}=-\int_{\gamma} \lambda_{1} \beta_{0}, \\
& \iint_{D_{0}^{2}} \beta_{0} \wedge \beta_{1}=\int_{\gamma} \lambda_{0} \beta_{1},
\end{aligned}
$$

where $\gamma=D_{1}^{2} \cap D_{0}^{2}$.

The integral (24) is simplified as

$$
H(f)=-\frac{1}{2} \int_{\gamma} \lambda_{0} \beta_{1}+\lambda_{1} \beta_{0} \text {. }
$$

This formula coincides with the formula (22). Theorem 6 is proved.

\section{Acknowledgement}

The author is grateful to V.P.Leksin for the explication of the results of the paper [1], and to S.A.Melikhov for discussions. The results was presented at International Conference "Nonlinear Equations and Complex Analysis" in Russia (Bashkortostan, Bannoe Lake) during the period since March 18 (arrival day) till March 22 (departure day), 2013. The author was supported in part by Russian Foundation of Basic Research Grant No. 11-01-00822.

\section{References}

1. JA Berrick, FR Cohen, YL Wong, J Wu (2006) Configurations, braids, and homotopy groups. J Amer Math Soc 19: 265-326.

2. Graham Ellis, Roman Mikhailov (2010) A colimit of classifying spaces, Advances in Math, 223: 2097-2113; arXiv: 0804.3581.

3. Mitchell A Berger (2001) Hamiltonian dynamics generated by Vassiliev invariants, J. Phys. A: Math. Gen. 34: 1363-1374.

4. VA Vassiliev (1994) Complements of discriminants of smooth maps: topology and applications, 2-d extended edition, Translations of Math. Monographs, 98 AMS, Providence, RI: 268. 